ACCEPTED MANUSCRIPT

\title{
Feature-based characterisation of signature topography in laser powder bed fusion of metals
}

To cite this article before publication: Nicola Senin et al 2017 Meas. Sci. Technol. in press https://doi.org/10.1088/1361-6501/aa9e19

\section{Manuscript version: Accepted Manuscript}

Accepted Manuscript is "the version of the article accepted for publication including all changes made as a result of the peer review process, and which may also include the addition to the article by IOP Publishing of a header, an article ID, a cover sheet and/or an 'Accepted

Manuscript' watermark, but excluding any other editing, typesetting or other changes made by IOP Publishing and/or its licensors"

This Accepted Manuscript is @ 2017 IOP Publishing Ltd.

During the embargo period (the 12 month period from the publication of the Version of Record of this article), the Accepted Manuscript is fully protected by copyright and cannot be reused or reposted elsewhere.

As the Version of Record of this article is going to be / has been published on a subscription basis, this Accepted Manuscript is available for reuse under a CC BY-NC-ND 3.0 licence after the 12 month embargo period.

After the embargo period, everyone is permitted to use copy and redistribute this article for non-commercial purposes only, provided that they adhere to all the terms of the licence https://creativecommons.org/licences/by-nc-nd/3.0

Although reasonable endeavours have been taken to obtain all necessary permissions from third parties to include their copyrighted content within this article, their full citation and copyright line may not be present in this Accepted Manuscript version. Before using any content from this article, please refer to the Version of Record on IOPscience once published for full citation and copyright details, as permissions will likely be required. All third party content is fully copyright protected, unless specifically stated otherwise in the figure caption in the Version of Record.

View the article online for updates and enhancements. 


\title{
Feature-based characterisation of signature topography in laser powder bed fusion of metals
}

Nicola Senin ${ }^{1,2}$, Adam Thompson ${ }^{1}$ and Richard Leach ${ }^{1}$

${ }^{1}$ Manufacturing Metrology Team, Faculty of Engineering, The University of Nottingham, NG7 2RD, UK

${ }^{2}$ Department of Engineering, University of Perugia, 06125, Italy

E-mail: nicola.senin@unipg.it

\begin{abstract}
The use of state-of-the-art areal topography measurement instrumentation allows for a high level of detail in the acquisition of topographic information at micrometric scales. The three-dimensional geometric models of surface topography obtained from measured data create new opportunities for the investigation of manufacturing processes through characterisation of the surfaces of manufactured parts. Conventional methods for quantitative assessment of topography usually only involve the computation of texture parameters; summary indicators of topographyrelated characteristics that are computed over the investigated area. However, further useful information may be obtained through characterisation of signature topographic formations, as more direct indicators of manufacturing process behaviour and performance. In this work, laser powder bed fusion of metals is considered. An original algorithmic method is proposed to isolate relevant topographic formations and to quantify their dimensional and geometric properties, using areal topography data acquired by state-of-the-art areal topography measurement instrumentation.
\end{abstract}

\section{Introduction}

The investigation of a manufacturing process through the signature it leaves on the fabricated surface plays an important role in process development and optimisation [1,2]. The topography of a manufactured surface results directly from the physical phenomena that take place during fabrication, and typically contains information useful to infer and reconstruct what happened. The investigation of the signature surface features left behind by a manufacturing process is, therefore, particularly valuable for those processes that are still at an early stage of industrialisation; such as additive manufacturing of metals via powder bed fusion [3-5].

Recent advances in areal topography measurement [6] allow a high level of detail in the acquisition of topographic information at micrometric and sub-micrometric scales. However, conventional topography data analysis and characterisation methods generally involve only the computation of areal texture parameters (in particular, the set of areal parameters defined in ISO 25178-2 [7, 8]). As such, most surface texture analyses are conceptually oriented towards capturing the properties of an entire measured region into a series of summary indicators (texture parameters). Feature-based parameters are present in ISO 25178-2, but they exclusively refer to very specific types of features (hills and dales, see $[7,9,10]$ ) which may not necessarily be able to address a wider array of characterisation needs, where the user may be interested in the identification and characterisation of surface topography formations of any shape and size [11]. An opportunity is, therefore, missed in fully exploiting the acquired topographic information, pertaining to individual topographic features [12].

In this work, laser powder bed fusion (LPBF) of metals is examined [13]. Initial attempts at the measurement and identification of topographic features present on LPBF surfaces are found in recent research by the authors [14] and elsewhere $[15,16]$. Here an approach is presented that allows for a comprehensive identification and characterisation of the most relevant signature topographic features of LPBF surfaces. An original, algorithmic approach to the automated identification and characterisation of the signature features is proposed, which can be applied 
to topography datasets normally obtainable using current state-of-the-art topography measurement instrumentation.

\section{Test case}

\subsection{Overview}

The test surface used in this work is the top surface of a Ti6Al4V sample (approximate size $40 \mathrm{~mm} \times 14 \mathrm{~mm} \times 10 \mathrm{~mm}$ ) fabricated via LPBF (Renishaw AM250 selective laser melting machine) using build settings optimised for the material. The top surface is selected for investigation in the as-built conditions, i.e. with no post-processing, to retain the topographic formations produced during the process, useful when investigating the process signature. Note that we appreciate that this research does not represent all types of LPBF surface produced with different build conditions, material types or finishing processes - the aim is to provide a toolbox of techniques that can be applied in a given manufacturing scenario.

The main topographic formations of a LPBF surface are shown in Figure 1. The largest features, weld tracks, are the direct result of the formation and solidification of the melt pool, as the laser beam moves across the powder bed [17]. Their irregular shapes depend on multiple phenomena, including heat exchange, interactions between source material and the laser beam, and the topography of the layer or layers underneath [18].

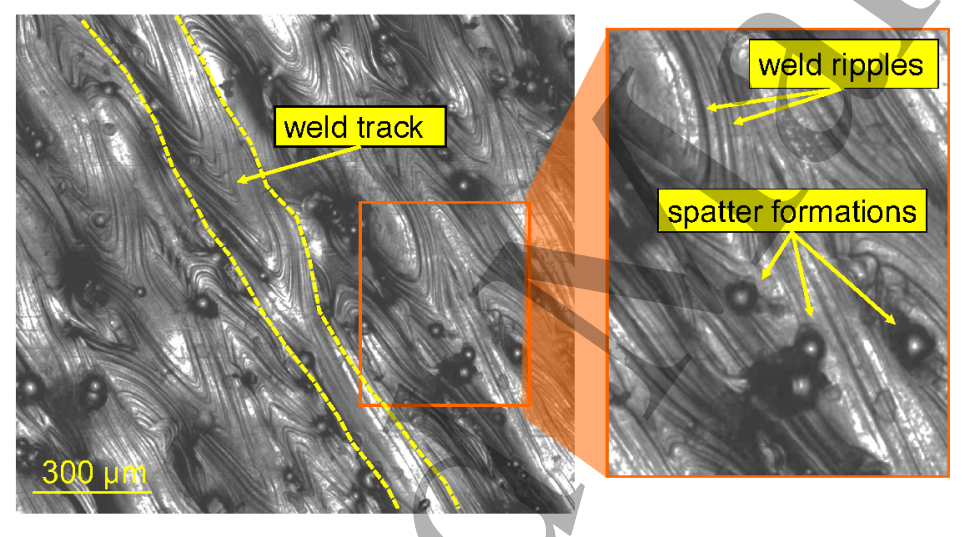

Figure 1. Main topographic formations visible on a LPBF surface (top layer). Focus-stacked image taken with a confocal microscope (field of view $(1.78 \times 1.78) \mathrm{mm}$, pixel dimensions $(0.625 \times 0.625) \mu \mathrm{m})$.

During the interaction between the laser beam and the powder bed, material is ejected from the melt pool; commonly referred to as spatter (Figure 1). Spatter may consist of unmelted or partially melted individual particles, agglomerates of particles, or droplets of molten material $[18,19]$. The shape and size of spatter formations depend greatly on the manufacturing process parameters and on the properties of the original particles [18]. For the test case, powder particles were between $10 \mu \mathrm{m}$ and $50 \mu \mathrm{m}$ in diameter and of quasi-spherical shape. Often, smaller particles are found attached to the larger particles, as is typical in LPBF [20]. Individual, unmelted particles typically retain their shape and size, though at smaller scales may show signs of mechanical impact with other particles. When partial melting occurs, spatter may appear as particle clusters, attached to each other by local surface welds. Complete melting leads to the formation of spatter in the form of molten droplets. These droplets retain quasi-spherical shape as a result of surface tension effects [18], but their sizes are generally larger than that of a source particle.

Recesses (see Figure 1) are either incomplete seams between neighbouring weld tracks (sometimes revealing portions of the layer underneath); or small cavities that form as a result of entrapped gaseous bubbles escaping from the material during processing [18]. Recesses due to open pores are generally much smaller than those produced by incomplete seams. Incomplete 
seams may not be present if the laser path is sufficiently dense, and/or the layer fabrication process is particularly well optimised.

A large-scale waviness component also typically encompasses the whole surface, affecting the straightness and regularity of the weld tracks on the surface. Many factors may contribute to the existence of the waviness component, including warping due to cooling effects during the process, as well as the topography of the layers underneath.

Finally, smaller-scale features are typically present on the LPBF surface, the most common of which is weld track ripples (chevron-shaped ripples overlaid onto the weld tracks, resúlting from the interaction between the laser and the melt pool - see Figure 1) [17]. At even smaller scales, cracks due to thermal cycles and local oxidation spots become visible (barely noticeable in Figure 1 due to insufficient resolution) [21] .

\subsection{Measurement set-up}

In this study, a total of four separate regions are measured, sufficiently far from the sample borders to be considered representative of "steady-state" manufacturing process conditions (i.e. avoiding unconventional thermal effects typical of edge regions). Each region is measured using a Zygo Newview 8300 coherence scanning interferometer (CSI) [22] and a 20× magnification objective (numerical aperture of 0.4). The measurement was performed with optimised source and detection settings as in [23]. The size of each measured region $(752.6 \times 752.6) \mu \mathrm{m}$, or $(1842 \times 1842)$ points, here referred to as field of view $(\mathrm{FOV})$, is obtained by stitching of $2 \times 2$ individual height images each of size $(420 \times 420) \mu \mathrm{m}$. The pixel size is $(0.409 \times 0.409) \mu \mathrm{m}$. Stitching was performed in Zygo's proprietary software, Mx.

\section{The feature-based characterisation pipeline}

\subsection{Overview}

The feature-based characterisation pipeline proposed in this work is comprised of dedicated methods and algorithms designed to specifically capture each target feature and its attributes. The dedicated pipeline is implemented in Matlab, though it should be possible to replicate the described methods in any other suitable software development environment; including through extensions of commercial surface metrology software. A detailed description of the featurebased characterisation pipeline is illustrated in the following sections; a diagrammatic overview is also provided in Appendix A1.

The feature-based characterisation pipeline targets spatter formations, weld tracks and weld ripples, and their attributes (for example, position, orientation, shape, size and density within the FOV). Larger-scale waviness and smaller-scale thermal cracks and oxidisation pools are not considered in this work. The class of surfaces being investigated has straight and parallel weld tracks; typically, the case for as-built horizontal layers generated by a LPBF process in raster/cross-hatch scanning mode [24].

\subsection{Pre-processing of the dataset}

Topography data is assumed to take the form of a height map (a matrix of height values distributed along the rows and columns of a regular $x y$ grid), as is generally the conventional output from current state-of-the-art commercial optical areal topography measurement instrumentation [6]. Data pre-processing consists of levelling, removal of non-measured points (voids) and removal of spike-like measurement artefacts. Levelling is implemented via leastsquares mean plane subtraction; voids are removed by replacement with weighted interpolation of valid neighbours [12]; and spike-like measurement artefacts are identified as local outliers and removed by interpolation of neighbours [25].

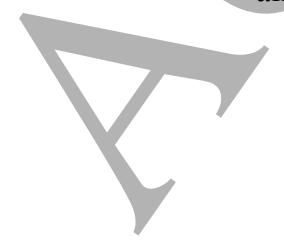




\subsection{Identification and characterisation of spatter}

The spatter identification algorithm used here is based on the assumption that spatter features are recognisable as protrusions from the surrounding surface. However, height-based discrimination is not possible, as spatter formations lay on top of an irregular surface, comprised of both the topography of the weld tracks and the large-scale waviness component mentioned in Section 2.1. To attenuate the latter, i.e. the large-scale underlying waviness, a Gaussian highpass filter with a $0.2 \mathrm{~mm}$ cut-off (also referred to as "nesting index" in ISO 25178-2 [7]) is applied (Figure 2). The chosen cut-off is based on previous visual assessment of the approximate width of the weld tracks (illustrated in Section 3.4). The high-pass filter is designed to preserve the local shape of the weld tracks (that is, its cross-section, and the shape of any smaller feature on top of it, e.g. particles, ripples, etc.), while at the same time flattening the substrate the tracks are laid onto, i.e. attenuating the vertical oscillations that weld tracks experience along their lengths. The chosen cut-off of $0.2 \mathrm{~mm}$ is, therefore, "calibrated" on the specific process set-up and is expected to vary with process variants.
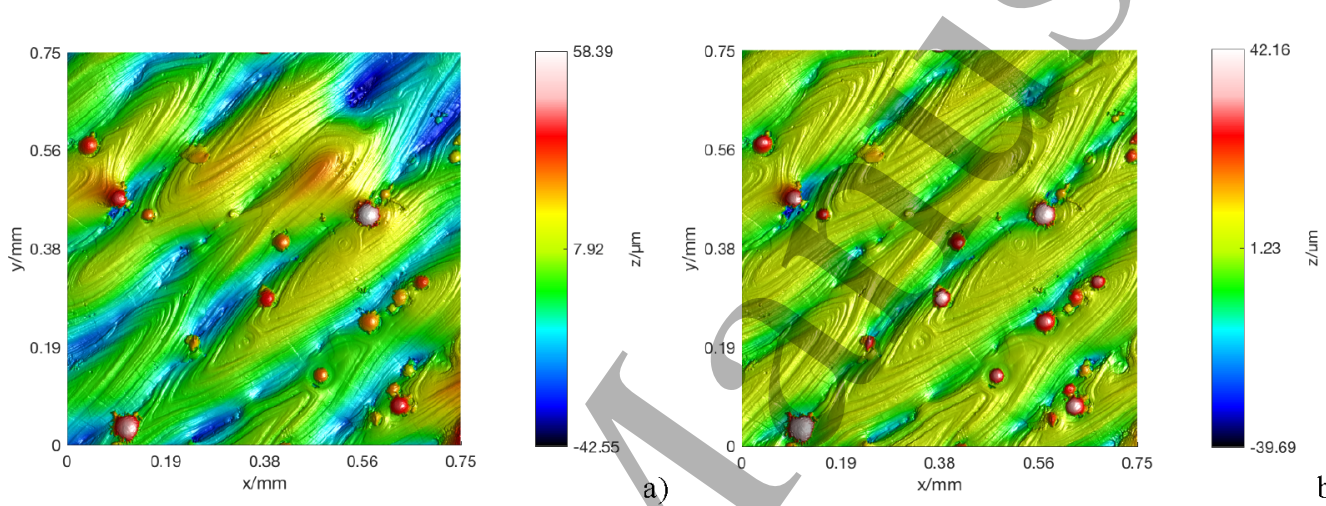

Figure 2. Removal of larger-scale waviness underlying the weld tracks (shown on the first of four CSI test datasets); a) original, after levelling, void and measurement artefact removal; b) high-pass filtered result.

On the high-pass filtered topography, sharp edges corresponding to spatter boundaries are recognised by means of an original approach referred to as cross-section contour stability analysis. In this approach, the topography is sectioned at different constant heights (threshold levels), and the most stable cross-sectional contours over a vertical range (i.e. those that change less between selected threshold levels) are isolated as steep boundaries, potentially belonging to spatter formations (see Figure 3a). Contour stability is assessed by collecting contours into a binned accumulation map; the size of the bins corresponding to the maximum allowed variation of "stable" contours. The most stable contours are extracted from the fullest bins in the accumulation map and identified as edges. Contour stability analysis is essentially an edgedetection method based on gradient thresholding [26], except that the gradient is specified indirectly through indication of maximum allowed displacement of the contour over a sequence of cross-sections.

The edges recognised by contour stability analy sis are then used to partition the height map, by means of the application of a sequence of morphological operators; more specifically: dilation and erosion (structuring element: disk of radius $0.8 \mu \mathrm{m}$ ) [26], followed by thinning (on $3 \times 3$ pixel windows [26]). The morphological operations are designed to trim the loose edges and close the small gaps in contour loops. Then, topologically disconnected regions are isolated by testing for topological connectedness on $3 \times 3$ pixel windows [26]. Regions are then filtered by size, aspect ratio and circularity, by computing binary image moments on the regions $x y$ footprints [26], to remove noise and discriminate between individual particles and clusters of particles; leading to the final spatter identification result (see Figure 3b). 

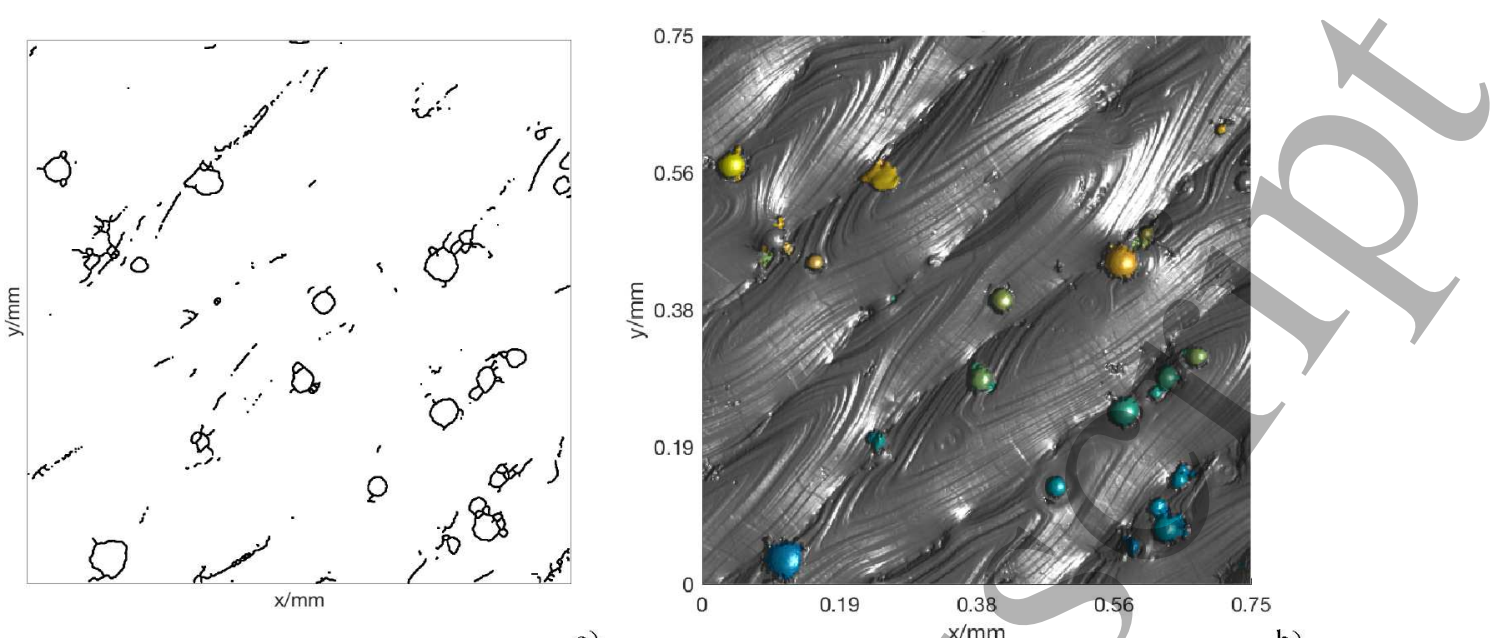

a)

Figure 3. Identification of spatter formations on the first CSI test dataset; a) edge map resulting from cross-section contour stability analysis (shown before the edge cleaning step); b) final segmentation result mapped onto the original topography (identified features are rendered in colour, the remaining topography is rendered in grey).

Some of the shape attributes used to identify spatter formations can also be used as a means of dimensional feature characterisation once features have been identified. However, it should be noted that the introduction of morphological operators to clean the edges in the selection mask during feature identification may slightly alter the position of the feature boundaries, ultimately propagating to the dimensional and geometric characterisation operations to follow. The strength of the morphological intervention should therefore carefully planned considering the effects on characterisation accuracy.

Definitions of size attributes for spatter formations are explained diagrammatically in Figure 4. The common subscript, $s$ (as in spatter), is used to name the attributes. Assuming that the $i^{\text {th }}$ feature has been identified in the dataset, its area $a_{\mathrm{s}, \mathrm{i}}$ is defined as the $x y$ area delimited by the $x y$ boundary of the feature, whilst the height of the feature $h_{\mathrm{s}, \mathrm{i}}$ is defined as the vertical distance between the average height of points located in the topmost region of the spatter formation and the average height of points surrounding the feature, following a conceptually similar approach to step height determination in the standard ISO 5436-1 [27]. Footprint area and height can be computed in the same manner for any type of spatter formation, i.e. individual particles, agglomerates and molten droplets.
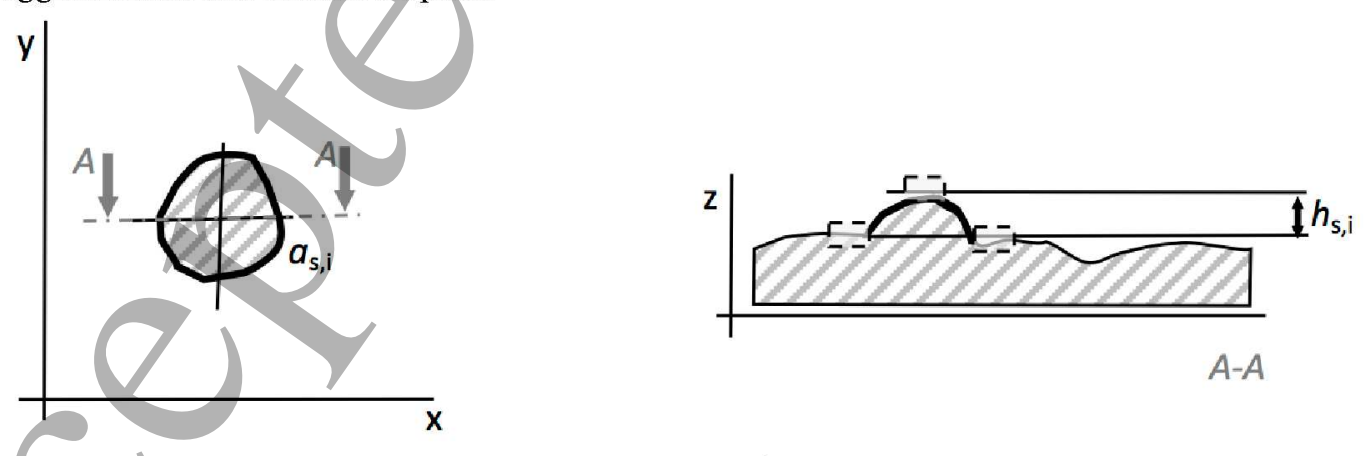

Figure 4. Geometric illustration of the attributes of the $i^{\text {th }}$ instance of a spatter feature; a) area $a_{\mathrm{s}, \mathrm{i}} ; \mathrm{b}$ ) height $h_{\mathrm{s}, 1}$. 
Figure 5. Spatter formations detected on a region of the test surface.

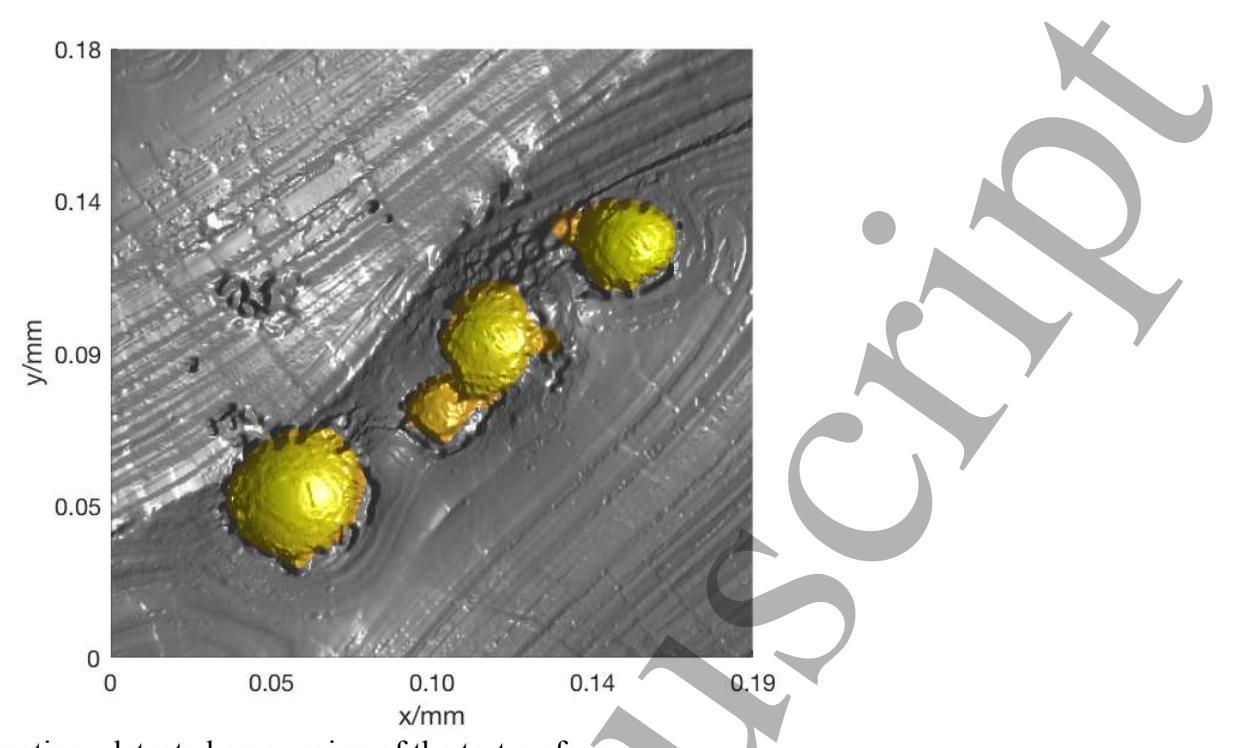

In Figure 5, an extracted region taken from the first test dataset is shown, highlighting the identification of three spatter formations. In Figure 6, the dimensional and geometric characterisation of the spatter formations shown in Figure 5 is illustrated. In Figure 6a, shape analysis via image-moments is applied to compute footprint area and aspect ratio for each individual spatter feature. In Figure 6b, the heights of each individual feature $h_{\mathrm{i}, \mathrm{s}}$ are shown along with the top and bottom regions used to compute them. In this application, the topmost region of the feature is algorithmically found by height thresholding, using the threshold value corresponding to the $10 \%$ areal material ratio $(\operatorname{Smr}(c)=10 \%$, see ISO $25178-2[7,28])$, evaluated on the areal material ratio curve computed for each individual formation. The use of the areal material ratio curve allows the definition of reference height value solely in terms of the percentage of material laying above/below it, and thus it is very robust to local shape irregularities. The bottom region is found by creating a selection mask defined as the set difference between the feature expanded areal footprint (morphological operator: dilate, structuring element disk of radius $4.5 \mu \mathrm{m}$ ) and the original one. It should be noted that the morphological operator only applies to the selection mask and is not altering the underlying topography in any way.

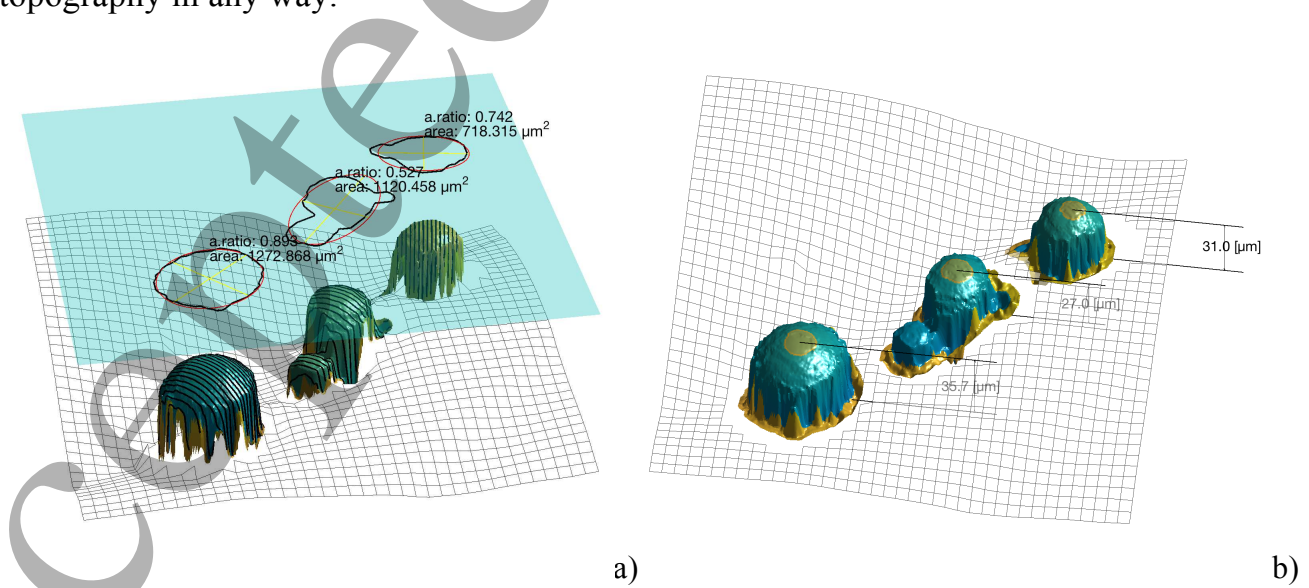

Figure 6. Dimensional and geometric characterisation of spatter formations: a) areal attributes via image moments, including footprint area $a_{\mathrm{i}, \mathrm{s}}$ calculated on the feature footprints; b) height $h_{\mathrm{i}, \mathrm{s}}$ calculated as vertical distances between topmost and surrounding feature regions (highlighted in yellow). 
3.4 Identification and characterisation of the weld tracks

Identified spatter features are removed from the high-pass filtered topography by using the mask that results from spatter feature identification. The void regions created by spatter removal are then filled by weighted interpolation of valid neighbours [25]. The resulting topography is subjected to a Gaussian pyramid decomposition [29,30] (four levels, dyadic decomposition, sigma of the Gaussian kernel: 2 pixels) to obtain a smoothed representation of the overall weld track shape. The fourth level of the pyramid decomposition is extracted and fitted to a smooth surface by weighted, non-parametric local regression to a second order polynomial surface (LOESS fitting [31]); as shown in Figure 7a. The LOESS fitting result is then upsampled to the original FOV resolution using bicubic resampling [26]. ISO morphological segmentation into hills (ISO 25178-2 [7, 28]) with $5 \% \mathrm{Sz}$ Wolf pruning and no area pruning leads to the partitioning shown in Figure $7 \mathrm{~b}$, where the partitioning result (also referred to as classification map, or segmentation map - essentially a multi-valued mask) is shown as a coloured overlay added on top of the original topography dataset (the one previously illustrated in Figure 2a).

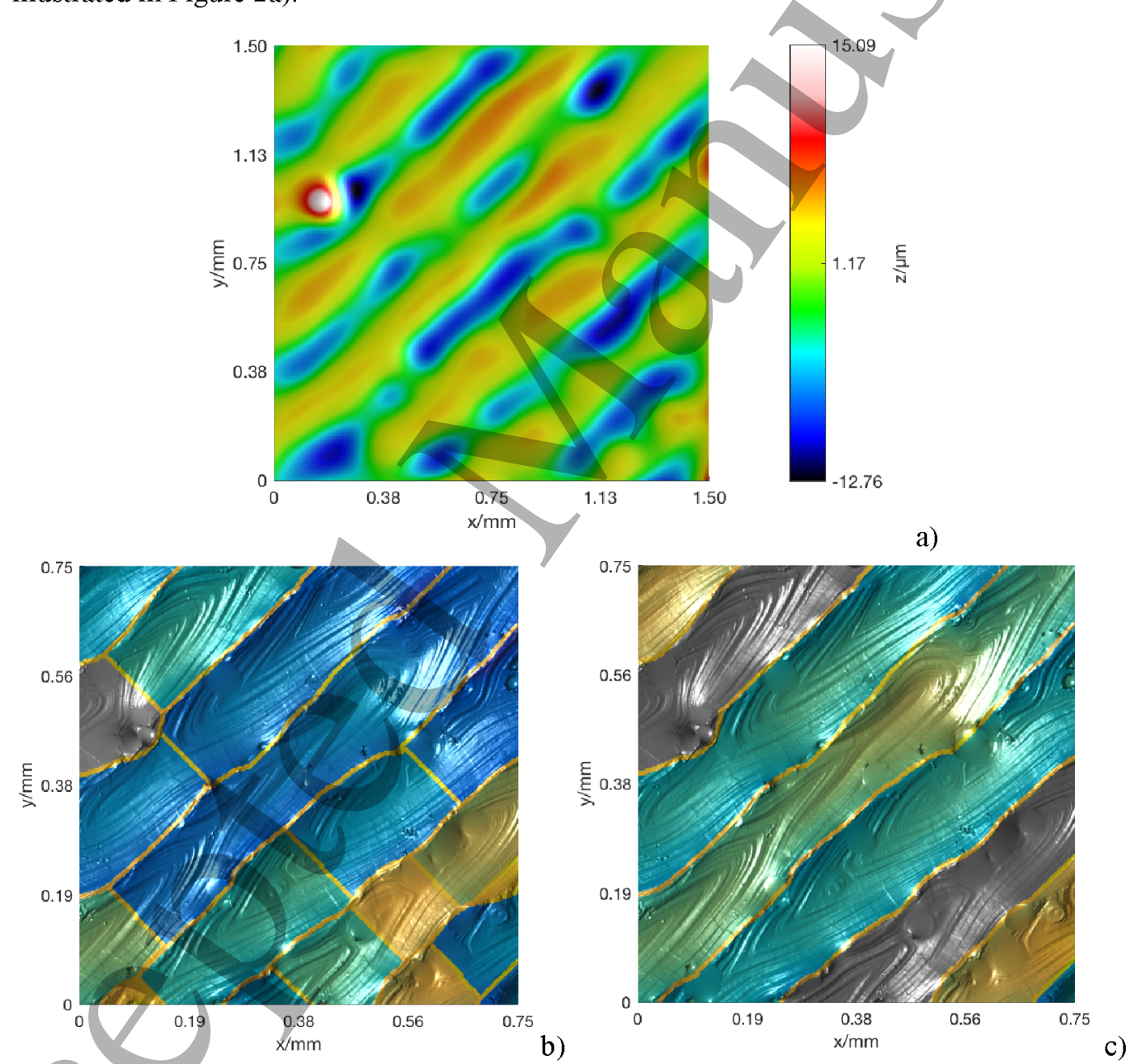

Figure 7. The weld track identification process; a) result of LOESS fitting; b) ISO morphological segmentation into hills; c) segment merging through examination of centroid alignment along the track direction.

Both pre-processing (pyramid decomposition, LOESS fitting and upsampling) and $S z$ Wolf pruning are designed to reduce the risk of over-partitioning, a known problem with ISO morphological segmentation, since each individual irregularity in the topography, no matter how small, may lead to the identification of an undesired, separate segment. Pre-processing reduces such risk by smoothing the topography (essentially removing weld ripples and other 
small singularities which could be identified as separate segments); pruning on the contrary acts on the partitioning result, by merging smaller segments into larger ones [9].

Despite pre-processing and pruning, the final weld tracks may still appear as split across multiple segments as shown in Figure $7 \mathbf{b}$. This is the result of residual depressed regions along the length of the weld tracks. However, segments can be merged by grouping in the direction of the weld tracks (based on centroid alignment), which in this test case, results in the segmentation shown in Figure 7c. Grouping based on centroid alignment is performed directly on the segmentation map, with no alteration to the original topography.

Once weld tracks have been identified within the FOV, orientation, width and height can be defined. Size attributes used to characterise weld tracks are explained diagrammatically in Figure 8 (the subscript $w t$ is assigned to weld track attributes). The orientation of the $i^{\text {th }}$ weld track $o_{\mathrm{wt}, \mathrm{i}}$ is the angle between the weld track axis and the $x$ axis. The weld track axis is found as the major axis of the equivalent ellipse computed via image moments on the weld track $x y$ footprint. The width of the $i^{\text {th }}$ weld track $w_{\mathrm{wt}, \mathrm{i}}$ is the mean distance between track boundaries, computed orthogonally to the track axis. Similarly, the height of the $i^{\text {th }}$ weld track $h_{\mathrm{wt}, i}$ is defined as the vertical distance between the average height of points located in the topmost region of the track and the mean height of points located at the track boundaries. The weld track axis is obtained by fitting an equivalent ellipse to the identified track footprint.

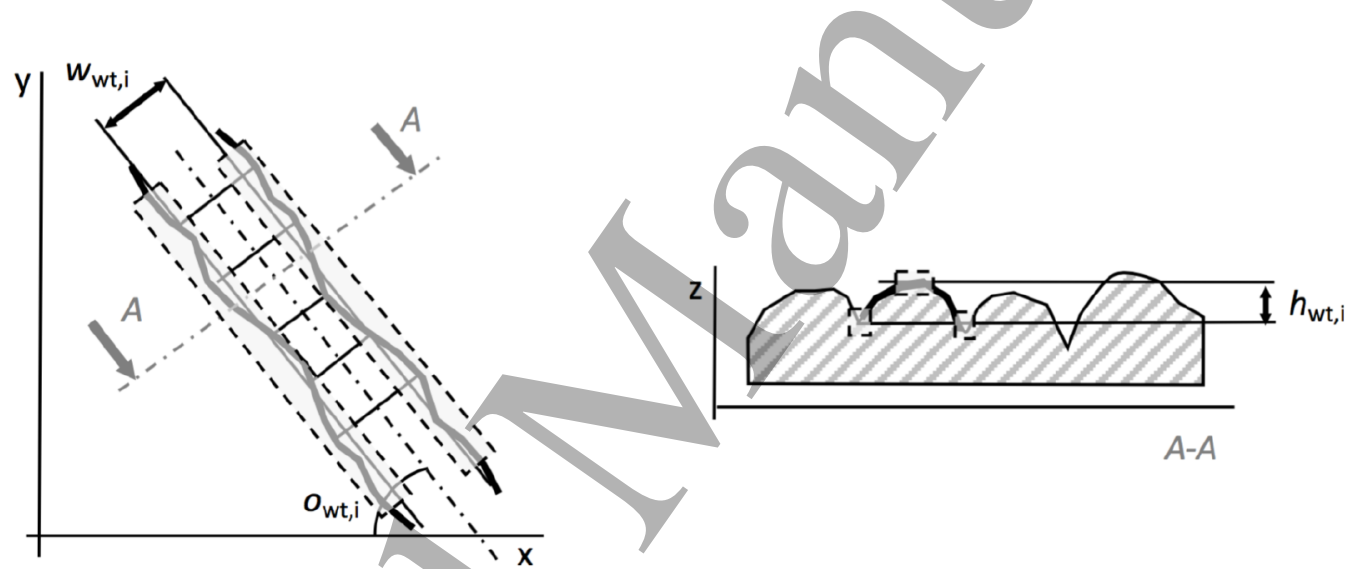

Figure 8. Geometric illustration of the attributes of the $i^{\text {th }}$ instance of a weld track; a) orientation $o_{\mathrm{wt}, \mathrm{i}}$ and width $w_{\mathrm{wt}, \mathrm{i}}$ b) height $h_{\mathrm{wt}, \mathrm{i}}$.

An example result of algorithmic computation of width as a function of length is shown in Figure 9, for one of the weld tracks visible in the same test dataset used in previous figures. Only tracks that are significantly represented within the FOV can be processed this way, whilst tracks significantly cropped at the image boundaries do not provide sufficient information to allow characterisation.
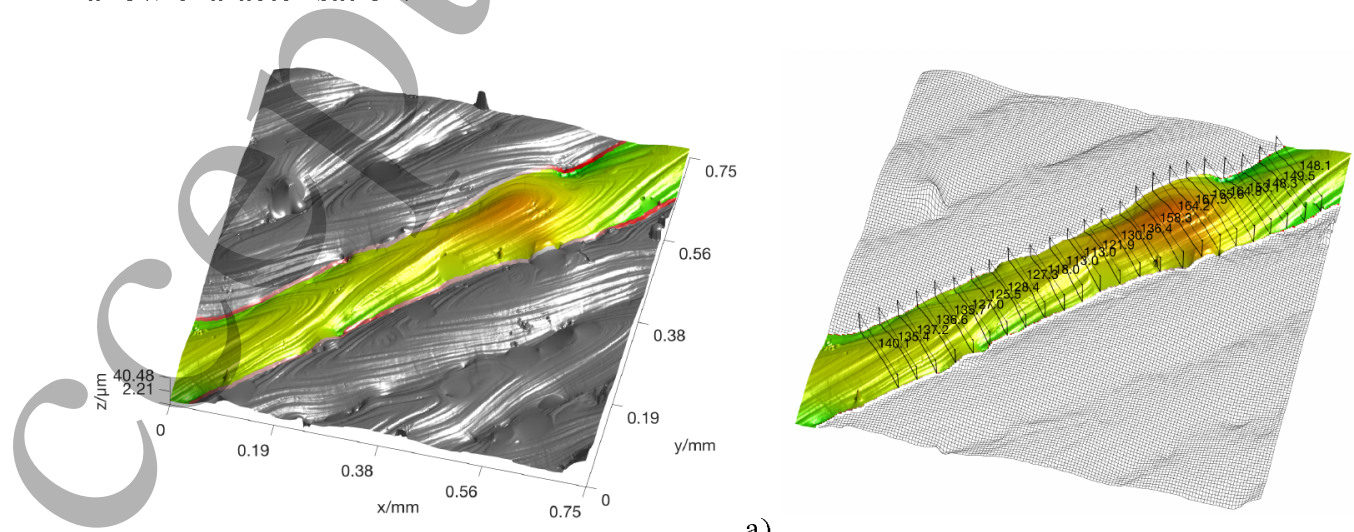

a)

b)

Figure 9. Results of the algorithmic computation of the width of one of the weld tracks visible in the dataset; a) selection; b) isolation of the feature and width computation on multiple cross-sections. 
As in width computation, the height of each weld track is computed at multiple cross-sections. At each, the top reference region is identified by the height threshold $\operatorname{Smr}(c)=10 \%$ (evaluated on the areal material ratio curve computed for the specific cross-section), whilst the bottom region is defined by the points located at the sides of the track. Mean values for the heights of the points belonging to the top and bottom regions are computed, and their differences are stored as local height values of the weld track at each cross-section.

\subsection{Identification and characterisation of the weld ripples}

Weld ripples can be studied in regions free of spatter. Potentially interesting target attributes are related to how ripples are distributed on the weld track (orientation, shape) and spacing. The region shown in Figure 10a has been extracted from one of the datasets. To remove the underlying shape of the weld tracks and thus isolate the ripple texture, a three-step process is applied: 1. a low-pass Gaussian convolution filter is applied with a cut-off of $40 \mu \mathrm{m}$ to identify the underlying large-scale topography and remove it (i.e. the residual is kept). 2. a smoothed approximation of the residual is obtained by LOESS fitting (span: $0.02 \%$ of the extents of the FOV, first order polynomial [31]) and also removed from the residual. The reason a second order polynomial is not needed for the LOESS fitting in the weld ripple case (used previously for weld tracks) is the small scale of the FOV, where the fitting is applied. An example result of the form removal process is shown in Figure $10 \mathrm{~b}$.

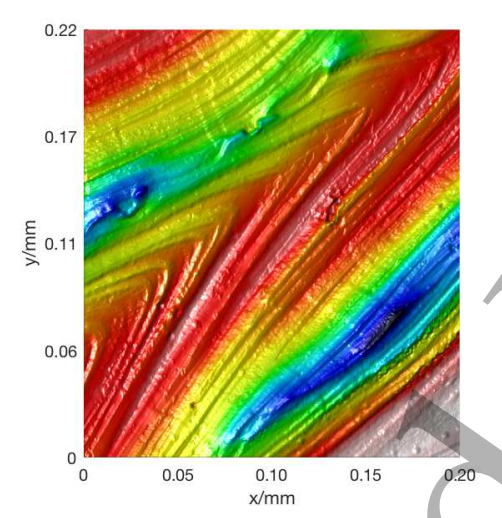

b)

Figure 10. Form-removal operation to remove weld track topography and highlight the weld ripples; a) original, levelled topography (height-based colouring); b) residual topography after high-pass Gaussian filtering and subtraction of the LOESS fit smoothed approximation (height-based colouring).

The topography remaining after form removal can then be subjected to edge detection via the Canny algorithm [26], which leads to multiple detected edges, some of which represent ripple crests and valleys (see Figure 11a). Local edge orientation is then found by applying principal components analysis within a moving window of $5 \times 5$ pixels to determine local main texture direction (Figure 11b). 

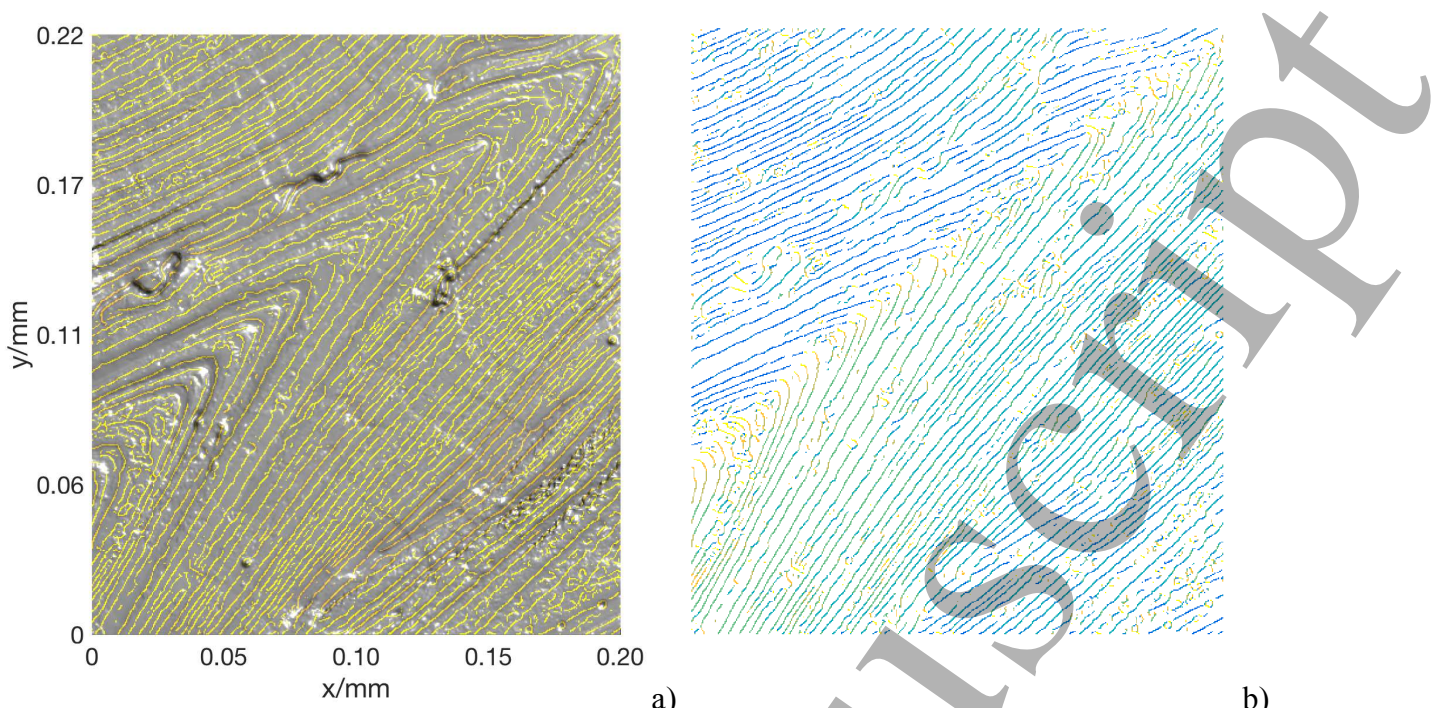

a)

Figure 11. Edge detection after form removal; a) results of the Canny algorithm; b) edges coloured according to local direction.

In order to compute the spacing between adjacent ripples, k-means clustering segmentation on local edge orientation [32] is applied to isolate the largest set of edges with similar orientation (Figure 12a). Selected edges are sectioned orthogonally to their mean direction, and a reference edge spacing measure is computed by computing the mean of the Euclidean distances of neighbouring intersection points laying along the same cross-section line (Figure 12b). As, typically, two crests are separated by a valley (i.e. amounting to three consecutive edges), ripple spacing is assumed as twice the edge reference spacing measure. Closer examination of Figure $12 \mathrm{~b}$ shows that not all intersections are reported by the algorithm. This failure is partly imputable to a hardcoded threshold on the maximum considerable distance between consecutive edges (case-specific, and computed based on initial observation of the results), and partly imputable to rounding error in the segment-to-segment intersection algorithms. This finding raises the issue of the importance of considering algorithmic error in the feature-based characterisation result and associated uncertainty, as further discussed in Section 5.
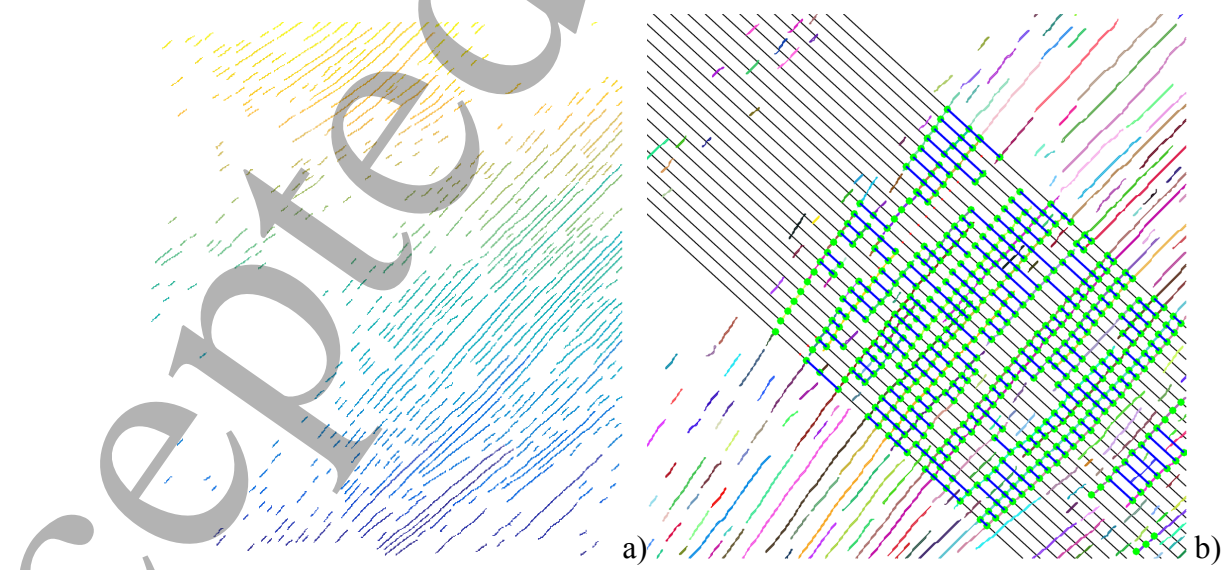

Figure 12. Characterisation of weld ripple spacing: a) edges with similar orientation extracted via clustering ( $\mathrm{k}$-means, $\mathrm{k}=8)$; $\mathrm{b}$ ) magnified view showing the local computation of ripple spacing as the distance between adjacent crests (green dots: intersection points between edges and cross sectioning lines; blue segments: valid samples for reference edge spacing computation).

3.6 Validation method

Validation of the proposed approach would, in theory, imply comparison to a reference procedure for feature-based characterisation of LPBF surfaces. However, there is no such 
procedure, and in general, for any surface topography, there is no established method of algorithmically identifying and characterising individual topographic features.

In this work, therefore, the results obtained by means of the proposed methods are compared to visual identification and manual measurement performed on the digital topography dataset through interactive functionality provided by commercial surface metrology software. MountainsMap by DigitalSurf [33] is used. The area of the $i^{\text {th }}$ spatter feature $a_{\mathrm{s}, \mathrm{i}}$ is obtained by drawing a closed contour around the visually identified feature. Height $h_{\mathrm{s}, \mathrm{i}}$ is similarly obtained by first tracing two closed contours, one delimiting the top region of the formation, the other delimiting its immediate surroundings. $h_{\mathrm{s}, \mathrm{i}}$ is then computed as the difference between two horizontal planes obtained by averaging the height values of points located within the traced regions. The width of the $i^{\text {th }}$ weld track $w_{\mathrm{wt}, \mathrm{i}}$ is obtained by drawing two parallel straight lines approximately following valleys either side of the track. The distance between the two lines is then taken as $w_{\mathrm{wt}, \mathrm{i}}$. Lines are always drawn in pairs around each weld track, visually averaging irregular track boundaries. Weld track orientation $o_{\mathrm{wt}, \mathrm{i}}$ is the orientation of the pair of parallel lines previously drawn for computing $w_{\mathrm{wt}, \mathrm{i}}$, measured with respect to the $x$ axis. To determine track height, three closed contours are drawn: one to identify the top of the $i^{\text {th }}$ weld track, and two regions at its sides. The height of the $i^{t h}$ track $h_{\mathrm{wt}, \mathrm{i}}$ is the height difference between the mean height of the top region and the mean height of the two side regions, aggregated. Each side region is reused for the neighbouring track. Weld ripples are too numerous to make the manual computation feasible, though sampled measurements of spacing are taken by drawing a pair of parallel lines along two consecutive ripple ridges and measuring the distance between these lines.

\section{Results}

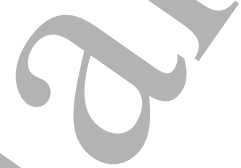

In Table 1, the performance of algorithmic and visual feature identification methods is shown, expressed in terms of the number of features detected on each topography dataset. Feature numbers could only be computed for spatter formations and weld tracks, as it was not possible to isolate individual weld ripples. Results were computed on the four test datasets obtained as described in Section 2.2.

Table 1. Number of identified features in the four datasets

\begin{tabular}{|c|c|c|c|c|}
\hline \multirow{2}{*}{ Test dataset ID } & \multicolumn{2}{|c|}{ Number of identified weld tracks } & \multicolumn{2}{c|}{ Number of identified spatter formations } \\
\cline { 2 - 5 } & Algorithmic & Visual & Algorithmic & Visual \\
\hline 1 & 8 & 7 & 23 & 22 \\
\hline 2 & 7 & 6 & 36 & 23 \\
\hline 3 & 8 & 6 & 38 & 14 \\
\hline 5 & 8 & 6 & 24 & 19 \\
\hline
\end{tabular}

The discrepancies in the number of identified weld tracks shown in Table 1 are due to different choices made by the algorithm and the operator, in relation to how to handle track instances barely appearing at the boundary of each dataset. Such instances are automatically included by the identification algorithm, but are discarded later at the characterisation stage because they lack sufficient information. In this case, they were immediately discarded by the operator and thus not included in the results of visual identification.

Concerning the number of spatter formations, the algorithmic method appears to be consistently returning a higher number of feature instances than the visual method. Further investigation of the results suggests that the operator discarded some of the smaller formations, interpreting them as noise or finding them to be visually indiscriminable from the background topography.

In Figure 13, areas and heights of mutually recognised spatter formations acquired using the manual and algorithmic procedures are compared through the computation of confidence intervals (CIs) for the population mean (data collected by merging the results for all the feature 
instances identified in the four datasets - the number of instances is reported in Table 1). In Figure 14, the same comparison is shown for widths and heights of the mutually recognised weld tracks.
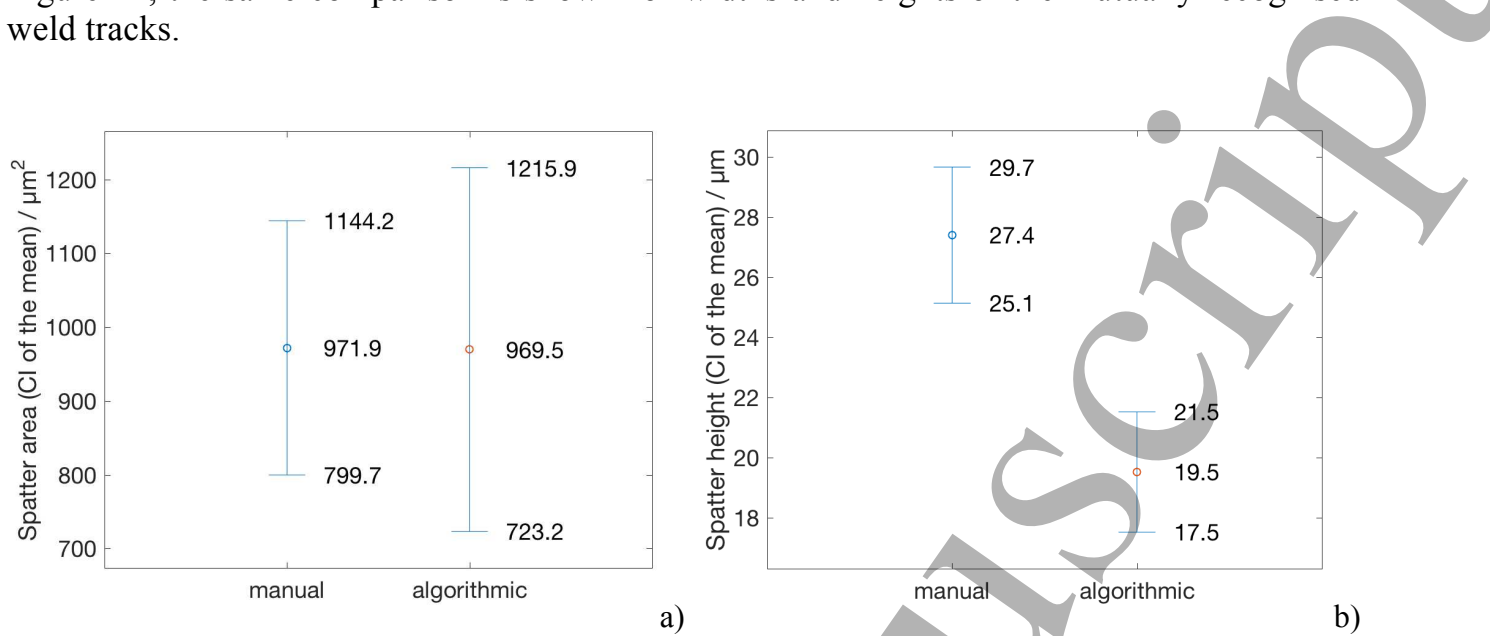

a)

Figure 13. Spatter formations - area and height: confidence intervals on the mean at $95 \%$ confidence for manual and algorithmic methods; a) population mean of $a_{s}$ (spatter area); b) population mean of $h_{\mathrm{s}}$ (spatter height). Sample obtained by aggregating all feature instances found in the four test datasets.

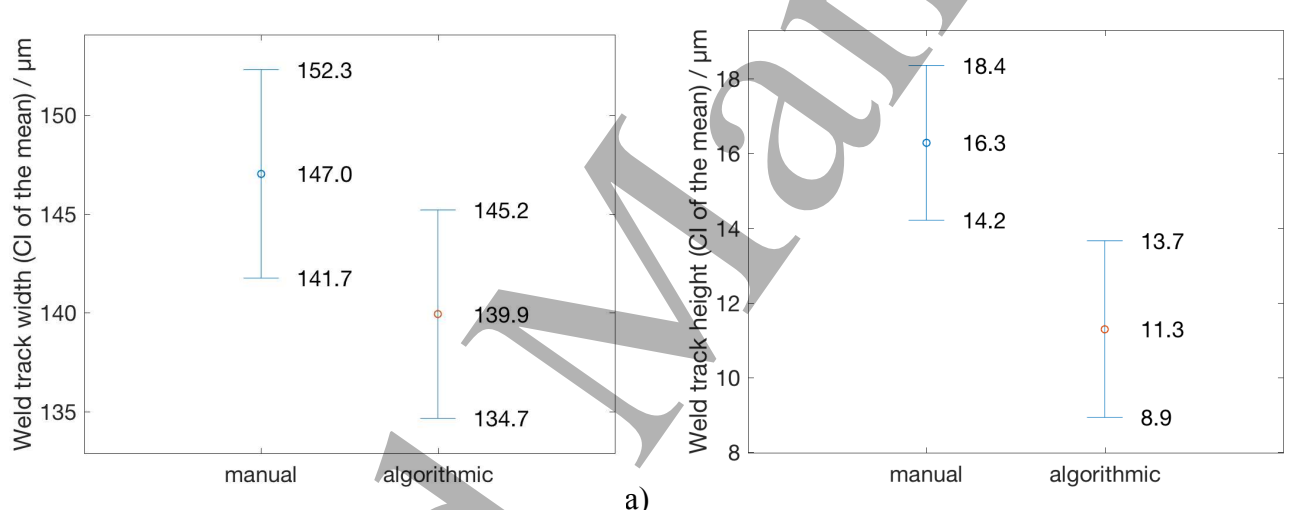

Figure 14. Weld tracks - width and height: confidence intervals on the mean at $95 \%$ confidence level for manual and algorithmic methods; a) population mean of $w_{\mathrm{wt}}$ (weld track width); b) population mean of $h_{w t}$ (weld track height). Sample obtained by aggregating all feature instances found in the four test datasets.

Whilst value dispersion is similar between visual and algorithmic methods (see CI widths in Figure 13 and Figure 14), the differences between the means obtained from algorithmic and manual methods are more significant. This is to be expected, given that attributes are computed differently between the two methods, essentially assigning different meanings to the same name. This is a relevant issue which will be discussed in Section 5.

In Figure 15, the results of manual and algorithmic computation of weld ripple spacing are shown. While the means are similar, the CI width for the manual method is much higher. This effect is due to both the smaller number of samples that can be collected by an operator in a reasonable time (five samples per dataset as opposed to approximately 400 samples per dataset in the algorithmic method), as well as the difficulties associated with reliable visual identification of weld ripple boundaries. 


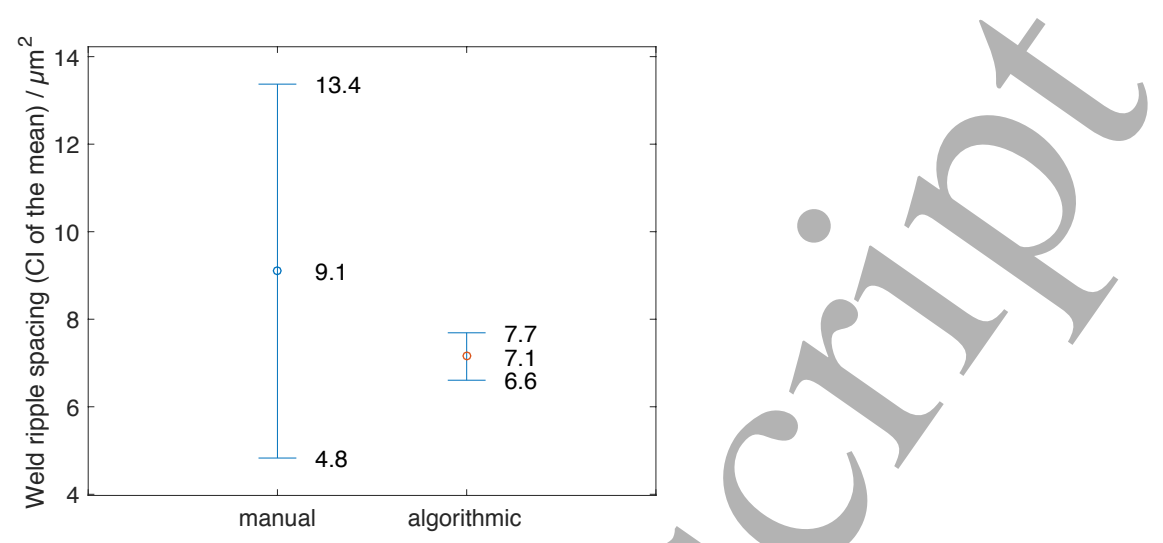

Figure 15. Weld ripple spacing: manual and algorithmic methods. Sample obtained by aggregating all feature instances found in the four test datasets.

\section{Discussion}

\subsection{Manual against algorithmic feature-based characterisation}

Feature-based characterisation based on visual identification and manual (computer-assisted) calculation of dimensional attributes is likely more flexible than the algorithmic method, given that it is performed by a human operator. However, the manual method is intrinsically less repeatable, its performance worsening with an increasing number of feature instances in the field of view. Moreover, the manual method is significantly less reproducible: as feature shapes become more complex and boundaries more difficult to ascertain, apparently straightforward concepts, such as width and height, become more difficult to define, and subjective interpretation on how feature dimensions should be cálculated becomes a factor of concern. Visual identification methods are similarly burdened, and as features become more complex and less clearly distinguishable from the background, it is increasingly difficult to capture the reasoning process followed by an expert operator in the assessment of whether or not a particular region of the topography should be identified as a feature instance.

Conversely, algorithmic approaches require greater initial investment to set-up. Moreover, the complexity of some feature shapes and their surroundings may render the endeavour of automated identification prohibitively difficult in some circumstances. Additionally, stricter checks on the results and handling of special cases are almost invariably necessary to filter out incorrectly identified topographic formations. Nevertheless, it is undeniable that, once an appropriate algorithmic pipeline is in place, both for identification and for characterisation of the target features, regardless of associated assessment error, significant advantages can be seen both in terms of repeatability and reproducibility. In terms of repeatability, algorithmic methods are particularly advantageous in cases where large numbers of feature instances are expected. In terms of reproducibility, algorithmic methods are particularly advantageous because the definitions of 'what a feature is' and 'what a specific feature's attributes are' are implicitly cast in the procedure used to identify the feature and to compute its attributes.

\subsection{Generalising the feature-based approach}

The methods presented here have been "calibrated" for a specific test case, involving LPBF of metals. Different applications will likely require different set-ups of the main algorithmic data processing parameters, and in some cases may require entirely new solutions; specifically designed to overcome case-dependent challenges related to either feature identification, feature characterisation or both. Evidently, such a heavily-customised approach to feature-based characterisation requires a significant upfront overhead for identifying a suitable pipeline, and tuning it to the application-specific requirements. However, it is believed by the authors that this initial cost is balanced by the benefits discussed in the previous section, i.e. improved

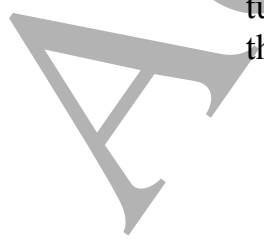


repeatability and reproducibility, (resulting from the removal of subjective assessment) and the capability to address a higher number of feature instances per dataset.

\subsection{Measurement uncertainty}

As for any measurement method, some estimation of associated uncertainty should be provided together with the results of feature-based characterisation. In principle, as the proposed solution essentially comprises a series of data processing steps, the assessment of measurement uncertainty consists of both understanding the error associated to the input data (i.e. to the topography datasets as measured by areal topography measurement instruments), and how the associated uncertainty propagates through the algorithmic data processing pipeline. Methods for investigating measurement error associated to areal topography datasets have been recently introduced in the literature [17, 34-36], though investigation of error propagation through the feature-based characterisation pipeline is still at the initial stages [37]. Algorithmic error in particular must be investigated in great detail, as two different implementations of the same conceptual approach, or even the same implementation, evolving over time, may lead to different results. The investigation of uncertainties associated to dimensional and geometric characterisation of localised topographic features represents one of the major challenges in further development of the feature-based characterisation paradigm.

\section{Conclusions}

An algorithmic approach has been presented for the automated identification and characterisation of signature features present on the surface of metallic parts fabricated using laser powder bed fusion. As opposed to describing surface topography through areal field texture parameters (such as those defined in the ISO 25178-2 standard), the proposed solution provides a customised data processing pipeline for extraction of information directly relating to the shape, size and position of topographic features of interest.

The proposed solution examines weld tracks, spatter formations and weld ripples, computing attributes which are important to manufacturing researchers interested in gaining further insight into the manufacturing process. The proposed method has been applied to four test datasets extracted from a Ti6Al4V sample surface manufactured by a selective laser melting machine. Results produced using the proposed method have been compared to those obtained by visual identification and manual measurement, performed with the assistance of commercial software. The proposed method provides a more repeatable and reproducible result, overcoming the drawbacks of subjective assessment typical of human operators However, the issue is raised that apparently simple concepts, such as width or height, may acquire completely different meanings when considering complex feature shapes, depending on the procedure adopted to compute them.

Texture parameters (e.g. those specified by ISO 25178-2) have the advantage that they can be calculated with little requirement for any prior knowledge of the surface (aside from that needed to set filter cut-offs and for form removal). By contrast, the feature-based characterisation approach illustrated in this work does indeed require prior knowledge about the shapes and sizes of the features that will be encountered (e.g. ballpark estimations for setting-up the method parameters). While evidently the need for knowledge is an obstacle to the ease of application and the generalisability of the method, feature-based characterisation provides a whole new set of opportunities for the development of more advanced data analysis pipelines.

Although the proposed procedure has been designed to address a very specific application, (i.e. the characterisation of signature topographic features in laser powder bed fusion metallic surfaces), the solution presented in this work provides a clear indication of the importance of feature-based characterisation as a new paradigm for surface metrology. The general outcome of this work is the demonstration that customised analysis pipelines can be built to directly 
address end-user information needs (for example, the desire to know the dimensional and geometric properties of specific features of interest), as opposed to simply computing a large array of summary indicators (i.e. ISO 25178-2 areal field texture parameters) which may not necessarily provide a direct answer to the matter being investigated, especially when links to processing parameters or final function are required.

\section{Acknowledgements}

AT and RKL would like to thank EPSRC (Grants EP/M008983/1 and EP/L01534X/1) and 3TRPD Ltd. for funding this work. The authors would like to thank DigitalSurf for providing the MountainsMap software, and Prof. Chris Tuck and Alex Gasper (Centre for Additive Manufacturing, University of Nottingham) for helpful discussions on the LPBF process.

\section{References}

[1] Thomas T R 2014 Roughness and function Surf. Topog.: Metrol. Prop. 2014001

[2] Benardos P G, Vosniakos G C 2003 Predicting surface roughness in/machining: a review Int. J. Mach. Tools Manufac. 43 833-44

[3] Sames W J, List F A, Pannala S, Dehoff R R, Babu S S 2016 The metallurgy and processing science of metal additive manufacturing Int. Mater. Rev. 61 315-60

[4] Leach R K 2016 Metrology for additive manufacturing Measurement + Control 49 132-

\section{5}

[5] Townsend A, Senin N, Blunt L A, Leach R K, Taylor J S 2016 Surface texture metrology for metal additive manufacturing: a review Prec. Eng. 46 34-47

[6] Leach R K 2011 Optical measurement of surface topography (Berlin: Springer)

[7] ISO 25178-2 Geometrical product specifications, (GPS) -- Surface texture: Areal -- Part 2: Terms, definitions and surface texture parameters (International Organization of Standardization: Geneva)

[8] Leach R K 2013 Characterisation of areal surface texture (Heidelberg: Springer Verlag)

[9] Scott P J 2004 Pattern analysis and metrology: the extraction of stable features from observable measurements Proc. R. Soc. Lond. A 460 2845-64

[10] Blateyron F 2013 Areal field parameters. In: Leach R K Characterisation of areal surface texture (Berlin: Springer)

[11] Senin N, Blunt L A, Leach R K, Pini S 2013 Morphologic segmentation algorithms for extracting individual surface features from areal surface topography maps Surf. Topog.: Metrol. Prop. 1 15005-

[12] Senin N, Blunt L A 2013 Characterisation of individual areal feaures In: Leach R K Characterisation of areal surface rexture (Berlin: Springer)

[13] Gibson I, Rosen D, Stucker B 2015 Additive manufacturing technologies (New York: Springer-Verlag)

[14] Thompson A, Senin N, Leach R K 2016 Towards an additive surface atlas Proc. ASPEleuspen 2016 Summer Topical Meeting - Dimensional Accuracy and Surface Finish in Additive Manufacturing, Raleigh, USA

[15] Lou S, Sun W, Zeng W, Abdul-Rahman H S, Jiang X, Scott P J 2017 Extraction of process signature features from additive manufactured metal surfaces Proc. $16^{\text {th }}$ Met. \& Props, Gothenburg, Sweden, Jun

[16] Reese Z, Evans C J, Fox J C, Taylor J S 2017 Observations on the surface morphology of laser powder bed fusion metal surfaces. Proc. $16^{\text {th }}$ Met. \& Props, Gothenburg, Sweden, Jun

[17] Senin N, Thompson A, Leach R K 2017 Characterisation of the topography of metal additive surface features with different measurement technologies Meas. Sci. Technol. 28095003

[18] Khairallah S A, Anderson A T, Rubenchik A, King W E 2016 Laser powder-bed fusion additive manufacturing: Physics of complex melt flow and formation mechanisms of pores, spatter, and denudation zones Acta Materialia 108 36-45 
[19] Simonelli M, Tuck C, Aboulkhair N T, Maskery I, Ashcroft I, Wildman R D, Hague R 2015 A study on the laser spatter and the oxidation reactions during selective laser melting of 316L stainless steel, Al-Si10-Mg, and Ti-6Al-4V Metallur. Mater. Trans. A 46 3842-51

[20] Liu Y, Yang Y, Mai S, Wang D, Song C 2015 Investigation into spatter behavior during selective laser melting of AISI 316L stainless steel powder Materials \& Design 87 797806

[21] Hirsch M, Catchpole-Smith S, Patel R, Marrow P, Li W, Tuck C, Sharples S D, Clare A T 2017 Meso-scale defect evaluation of selective laser melting using spatially resolved acoustic spectroscopy Proc.Roy. Soc. A: $\mathbf{4 7 3} 20170194$

[22] de Groot P 2011 Coherence scanning interferometry. In. Leach R K Optical measurement of surface topography (Berlin: Springer)

[23] Gomez C, Su R, Thompson A, DiSciacca J, Lawes S, Leach R K 2017 Optimization of surface measurement for metal additive manufacturing using coherence scanning interferometry Opt. Eng. 56111714

[24] Parry L, Ashcroft I A and Wildman R D 2016 Understanding the effect of laser scan strategy on residual stress in selective laser melting through/thermo-mechanical simulation Additive Manufacturing 12 1-15

[25] Senin N, Blunt L A, Tolley M 2012 The use of areal surface topography analysis for the inspection of micro-fabricated thin foil laser targets for ion acceleration Meas. Sci. Technol. 23105004

[26] Gonzalez R C, Woods R E 2002 Digital image processing (Prentice Hall)

[27] ISO 5436-1 Geometrical Product Specifications (GPS) -- Surface texture: Profile method; Measurement standards -- Part 1: Material measures (International Organization of Standardization: Geneva)

[28] Blateyron F 2013 Areal feature parameters. In: Leach R K Characterisation of areal surface texture (Berlin: Springer)

[29] Senin N, Leach R K, Pini S, Blunt L A 2015 Texture-based segmentation with Gabor filters, wavelet and pyramid decompositions for extracting individual surface features from areal surface topography maps Meas. Sci. Technol. 26095405

[30] Adelson E H, Anderson C H, Bergen J R, Burt P J, Ogden J M 1984 Pyramid methods in image processing $R C A$ Engineer 29 33-41

[31] Cleveland W S, Devlin S J 1988 Locally-weighted regression: an approach to regression analysis by local fitting J. Am. Stat. Assoc. 83 596-610

[32] Senin N, Ziliotti M, Groppetti R 2007 Three-dimensional surface topography segmentation through clustering Wear 262 395-410

[33] Digital Surf 2016 Digital Surf MountainsMap Software.

[34] Thompson A, Senin N, Giusca C and Leach R K 2017 Topography of selectively laser melted surfaces: A comparison of different measurement methods Ann. CIRP 66 543-6

[35] MacAulay G D, Giusca C L 2016 Assessment of uncertainty in structured surfaces using metrological characteristics Ann. CIRP 65 533-6

[36] Haitjema H 2015 Uncertainty in measurement of surface topography Surf. Topog.: Metrol. Prop. 3035004

[37] Musso G, Senin N, Galetto M and Leach R K Towards uncertainty in dimensional metrology of surface features for advanced manufacturing. Proc. 16th Int. euspen Conf., Nottingham, UK, Jun 63-64 